\title{
Design Rules for Lossy Mode Resonance Based Sensors
}

\author{
Ignacio Del Villar, ${ }^{1, *}$ Miguel Hernaez, ${ }^{1}$ Carlos R. Zamarreño, ${ }^{1}$ Pedro Sánchez, ${ }^{1}$ Carlos \\ Fernández-Valdivielso, ${ }^{2}$ Francisco J. Arregui, ${ }^{1}$ and Ignacio R. Matias ${ }^{1}$ \\ ${ }^{1}$ Electrical and Electronic Engineering Department, Public University of Navarre, \\ Campus Arrosadia s/n, 31006 Pamplona, NA, Spain \\ ${ }^{2}$ Sociedad de Desarrollo de Navarra (SODENA), Avenida Carlos III el Noble, 36, $1^{\circ}$ Dcha, \\ 31003 Pamplona, NA, Spain \\ *Corresponding author: ignacio.delvillar@unavarra.es
}

Lossy mode resonances can be obtained in the transmission spectrum of cladding removed multimode optical fiber coated with a thin-film. The sensitivity of these devices to changes in the properties of the coating or the surrounding medium can be optimized by means of the adequate parameterization of the coating refractive index, the coating thickness and the surrounding medium refractive index (SMRI). Some basic rules of design, which enable the selection of the best parameters for each specific sensing application, are indicated in this work.

OCIS codes: $310.0310,060.2370,260.5740$.

\section{INTRODUCTION}

Generation of resonances in the transmission spectrum with cladding removed multimode optical fiber (CRMOF) coated with a thin-film has been proved successfully in [1-5]. Light propagation through semiconductor cladded waveguides has been studied in [6], where attenuation maxima 
of the light propagating through the optical waveguide can be obtained for specific thickness values and at certain wavelengths or incidence angles. This is due to a coupling between waveguide modes and a particular lossy mode of the semiconductor thin-film [7]. Since the phenomenon occurs when the lossy mode of the thin-film is near cut-off, there are thin-film thickness values that lead to transmission attenuation maxima [6]. The same phenomenon can be observed if the variable is the wavelength and not the thickness. If the thin-film thickness is fixed, a resonance is visible in the electromagnetic spectrum for those incident wavelength values where there is a mode near cut-off in the overlay. These resonances are not surface plasmon resonances (SPR), but lossy mode resonances (LMR) $[1,8]$ or guided mode resonances [9] (depending on the expression used by the author). SPR occurs when the real part of the thinfilm permittivity is negative and higher in magnitude than both its own imaginary part and the permittivity of the material surrounding the thin-film [9]. LMR occurs when the real part of the thin-film permittivity is positive and higher in magnitude than both its own imaginary part and the material surrounding the thin-film [9]. Consequently, SPR can be obtained with materials with a high imaginary part of the refractive index (typically metals [10-11]), whereas LMR is achieved for materials with low imaginary part of the refractive index.

LMRs have been observed with metal oxides, such as ITO [1-2], $\mathrm{TiO}_{2}$ [3], and Indium Oxide [4]. Recently, polymers also have been proved successfully for the same purpose [5]. In addition to this, LMRs appear for both TM and TE polarized light and the generation of multiple resonances without modifying the optical fiber geometry is also possible [1]. Consequently, they constitute a promising research field that could compete with the largely established SPRs.

There is a number of recent publications with sensing applications based on lossy mode resonances: refractometers [1,3], where results overcome the sensitivity of LPFGs and are 
comparable to SPR based devices [3], humidity sensors [2], or even pH sensors [5]. So far, some rules for design have been given for the design of refractometers [4], but nothing has been told about the validity of these hints for a humidity sensor or a $\mathrm{pH}$ sensor yet. The aim of this article is to give some general rules that can help the designer to choose the adequate material for each specific application. To this purpose, devices based on the deposition of two different materials are compared: $\mathrm{TiO}_{2}$ and a polymeric matrix based on the combination of poly(allylamine hydrochloride) (PAH) and poly(acrylic acid) (PAA).

The remainder of this paper is organized as follows. In Section 2 it is presented the experimental setup and the fabrication procedure for the sensing devices. In section 3 it is indicated the method used for the analysis of the propagation of light trough the thin-film, and the modeling of $\mathrm{TiO}_{2}$ and PAH/PAA. Numerical results and rules for the design of LMR based sensors are given in section 4. Conclusions are included in Section 5.

\section{EXPERIMENTAL SECTION}

\subsection{Materials}

Titanium(IV) oxide nanoparticles of diameter $21 \mathrm{~nm}$ and the polymers poly(allylamine hydrochloride) (PAH) (Mw 100,000), poly(acrylic acid) (PAA) (Mw 35,000), and poly(sodium 4-styrenesulfonate) (PSS) (Mw 70000), were obtained from Sigma-Aldrich Inc. Aqueous solutions of PAH and PAA $(10 \mathrm{mM})$ were prepared using ultrapure deionized water $(18.2 \mathrm{M} \Omega)$ and adjusted to $\mathrm{pH}$ 4.4. The same procedure was used for the preparation of aqueous solutions of $\mathrm{TiO}_{2}(0.33 \%$ wt) and PSS $(10 \mathrm{mM})$, with the exception that both of them were adjusted to $\mathrm{pH}$ 2.0. The $\mathrm{pH}$ of the solutions was verified using an electronic $\mathrm{pH}-$ meter (Crison 
Inc.) and adjusted when necessary by adding a few drops of $\mathrm{NaOH}$ or $\mathrm{HCl}$. Plastic-clad silica fibers of 200/225 $\mu \mathrm{m}$ core/cladding diameter (FT200EMT) were provided by Thorlabs Inc.

\subsection{Deposition of the nanocoating}

The cladding of the optical fiber is removed in a portion of several centimetres in order to deposit a thin-film, which will support one or more lossy modes. After cladding removal the optical fibers were cleaned as described in [12].

The $\mathrm{TiO}_{2}$ coating was deposited onto the optical fiber core fragment by means of the Layer by Layer (LbL) method [13]. The PSS solution was used as anionic polyelectrolyte and the solution of $\mathrm{TiO}_{2}$ nanoparticles was used as cationic polyelectrolyte. A SEM image of the deposition of $\mathrm{TiO}_{2} / \mathrm{PSS}$ on optical fiber is shown in Fig. 1a.

The same LbL procedure was used for the build up process of the PAH/PAA coating. The PAA solution was used as anionic polyelectrolyte and the solution of PAH was used as cationic polyelectrolyte. A SEM image of the deposition of PAH/PAA on optical fiber is shown in Fig. $1 b$.

\subsection{Device characterization}

Transmission spectra were obtained by using the experimental setup of Fig. 2. A white light source Ando AQ-4303B, which covers a band of 400 to $1800 \mathrm{~nm}$, is connected to one end of the optical fiber. The other end is connected through a bifurcated optical fiber (VIS-NIR from OceanOptics with low $\mathrm{OH}$ content) to two spectrometers: a UV/Vis spectrometer (HR4000) and a NIR spectrometer (NIR512), both of them from Oceanoptics Inc. This permits to monitor a wavelength range from 400 to $1500 \mathrm{~nm}$. 
Since the spectrum of the white light source is not flat, the transmission spectra are referenced to a spectrum obtained before the deposition starts.

\section{THEORY}

The method used for simulating the propagation of light through a coated CRMOF is based on that used in [1]. In order to obtain the transmitted optical power in the setup of Fig.2 the attenuated total reflection (ATR) method with a Kretschmann configuration must be applied. In this way, the reflectivity as a function of wavelength and incidence angle is obtained at the coating - fiber core interface $\mathrm{R}(\theta, \lambda)[10,14]$, where $\lambda$ is the light wavelength and $\theta$ the angle of incidence. The expression for calculation of the transmitted optical power is $[10,14]$ :

$$
T(\lambda)=\frac{\int_{\theta_{c}}^{90^{\circ}} p(\theta) R^{N(\theta)}(\theta, \lambda) d \theta}{\int_{\theta_{c}}^{90^{\circ}} p(\theta)}
$$

where $\theta_{c}$ is the critical angle, $N(\theta)$ is the number of reflections at the coating - fiber core interface, and $p(\theta)$ is the power distribution of the optical source (a Gaussian distribution [11]). $R^{N(\theta)}(\theta, \lambda)$ represents the reflected light as a combination of the reflected power in TE and TM mode polarization [14].

The wavelength dependence of silica, which is the optical waveguide material, is expressed according to the Sellmeier expression [15]:

$$
n^{2}(\omega)=1+\sum_{j=1}^{m} \frac{B_{j} \omega_{j}^{2}}{\omega_{j}^{2}-\omega^{2}}
$$


With parameters: $\quad B_{1}=0.691663, \quad B_{2}=0.4079426, \quad B_{3}=0.8974794, \quad \lambda_{1}=0.0684043 \mu \mathrm{m}, \quad \lambda_{2}=$ 0.1162414 , and $\lambda_{3}=9.896161$, where $\lambda_{j}=2 \pi c / \omega_{j}$ and $c$ is the speed of light in vacuum.

The Lorentz model [16], is used for the dispersion of $\mathrm{TiO}_{2} / \mathrm{PSS}$ :

$$
\varepsilon(E)=\varepsilon_{\infty}+\sum_{k} \frac{A_{k}}{E_{k}^{2}-E^{2}-i B_{k} E}
$$

where $A_{k}, B_{k}$ and $E_{k}$ are respectively the amplitude, the center energy and the broadening of the $\mathrm{k}^{\text {th }}$ oscillator, $E$ is the photon-energy and $\varepsilon_{\infty}$ is an offset that indicates the permittivity at high frequency. For the sake of simplicity a single oscillator is used in expression (3). The parameters that lead to a best fit are: $\varepsilon_{\infty}=1, B_{k}=1.2 \mathrm{eV}, A_{k}=101 \mathrm{eV}^{2}$ and $E_{k}=6.2 \mathrm{eV}$. They are calculated by computing numerically the global error between the experimental and the theoretical transmission spectra for different coating thicknesses.

Finally, the wavelength dependence of PAH/PAA is obtained using the same procedure and formulation. The parameters obtained are: $\varepsilon_{\infty}=1, B_{k}=0.31 \mathrm{eV}, A_{k}=154.5 \mathrm{eV}^{2}$ and $E_{k}=11.5 \mathrm{eV}$. The dispersion curves of index of refraction of both $\mathrm{TiO}_{2} / \mathrm{PSS}$ and PAH/PAA are compared in Fig. 3.

\section{RESULTS}

\subsection{Generation of lossy mode resonances with TiO2/PSS and PAH/PAA}

A $\mathrm{TiO}_{2} / \mathrm{PSS}$ coating is deposited with $\mathrm{LbL}$ method on the cladding removed region of the multimode optical fiber. The same procedure is followed in the case of a device coated with PAH/PAA. 
The transmitted power is represented in Figs. 4 and 5 as a function of the coating thickness and wavelength for the two nanocoatings deposited: $\mathrm{TiO}_{2} / \mathrm{PSS}$ and PAH/PAA respectively. In both cases, the sensitive region is surrounded by ultrapure water (with a conductivity of $18.2 \mathrm{M} \Omega$ and a refractive index of 1.321 at $1300 \mathrm{~nm}$ ). Henceforward the acronym SMRI will be used for the surrounding medium refractive index.

The simulations agree with the experimental results, which confirms the validity of the model of section 3. The regions in black represent low transmission, which indicates the presence of a lossy mode resonance (LMR)

In order to observe the shape of the collected spectra, in Fig. 6, two spectra obtained from devices with $\mathrm{TiO}_{2} / \mathrm{PSS}$ coatings of thickness 333 and $1165 \mathrm{~nm}$ are represented. Additionally, two spectra obtained for devices with PAH/PAA coatings of thickness $750 \mathrm{~nm}$ and $1200 \mathrm{~nm}$ are also shown in Fig. 7. Both for Fig. 6 and Fig. 7 the SMRI is 1.321.

The sensitivity to different parameters of the thin-film coated CRMOF will be analyzed in detail in the next subsections.

For the sake of simplicity the following notation will be used:

Sthickness: sensitivity to variations of coating thickness expressed as wavelength shift vs thickness variation $(\mathrm{nm} / \mathrm{nm})$.

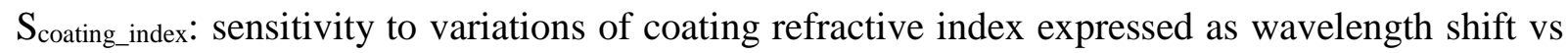
refractive index variation (nm/RIU). RIU means refractive index unit.

SSMRI: sensitivity to variations of SMRI expressed as wavelength shift vs refractive index variation (nm/RIU). RIU means refractive index unit.

\subsection{Sensitivity to coating thickness variations}


In Fig. 8 it is represented both theoretically and experimentally the evolution of the resonance wavelengths of the LMRs generated with $\mathrm{TiO}_{2} / \mathrm{PSS}$ and PAH/PAA coatings as a function of the coating thickness with SMRI fixed to 1.321 .

From Fig. 8 it is easy to compare both structures. First, we can observe that the number of LMRs obtained with $\mathrm{TiO}_{2} / \mathrm{PSS}$ is higher than the number of LMRs obtained with PAH/PAA. The reason is that the refractive index of $\mathrm{TiO}_{2} / \mathrm{PSS}$ structure is higher than the one of PAH/PAA (see Fig. 3a) and, hence, the $\mathrm{TiO}_{2} / \mathrm{PSS}$ structure can support more lossy modes than the PAH/PAA structure under the same conditions (thickness and SMRI).

Second, the plots of the LMRs are almost linear, which indicates that $S_{\text {thickness }}$ (the sensitivity to thickness expressed as wavelength shift versus coating thickness variation) remains constant for different thickness values of the coating.

In addition to this, it can be observed that $\mathrm{S}_{\text {thickness }}$ increases progressively as we move towards the first LMR in both cases ( $\mathrm{TiO}_{2} / \mathrm{PSS}$ and PAH/PAA coatings). In fact, if we compare the second LMR generated with the PAH/PAA coating with the seventh one obtained with the $\mathrm{TiO}_{2} / \mathrm{PSS}$ coating, both of them fit almost exactly, which proves that LMRs plotted as a function of the coating thickness occupy the same positions if they show the same $S_{\text {thickness, }}$ provided the SMRI remains constant. Moreover, a careful analysis of Fig. 8 permits to conclude that all plots converge in the same point of the wavelength axis, which is the cutoff wavelength of the coating waveguide.

In view of the differences between the results obtained with $\mathrm{TiO}_{2} / \mathrm{PSS}$ and PAH/PAA coatings, it is interesting to analyze $S_{\text {thickness }}$ as a function of the coating refractive index for a fixed SMRI of 1.321 (see Fig. 9a). In order to avoid the influence of wavelength, $\mathrm{S}_{\text {thickness }}$ is evaluated as the 
thickness variation that leads to a resonance wavelength shift between 700 and $800 \mathrm{~nm}$. The same is true for the analysis of Fig. 9b, which will be analyzed later.

After seeing the results of Fig. 8 and Fig. 9a, it can be concluded that in order to increase $\mathrm{S}_{\text {thickness, }}$, the best choice is to monitor the first LMR and to select a coating with high refractive index.

Another interesting observation in Fig. 9a is that the $\mathrm{S}_{\text {thickness }}$ ratio between the LMRs remains constant independently of the value of the coating refractive index.

In Fig. 10 the evolution of the resonance wavelength of the LMRs, generated with a coating with refractive index that of $\mathrm{TiO}_{2} / \mathrm{PSS}$ (see Fig. 3a), is represented as a function of thickness for two different SMRIs: 1.321 and 1.421.

It is obvious that $S_{\text {thickness }}$ is improved for all LMRs when the SMRI is increased. In order to obtain a better analysis, the evolution of $S_{\text {thickness }}$ as function of the SMRI for a coating with refractive index that of $\mathrm{TiO}_{2} / \mathrm{PSS}$, is plotted in Fig. $9 \mathrm{~b}$.

Here, it is interesting to observe that even though, as in Fig. 9a, there is an increase as a function the SMRI, the shape of the plots is different. Particularly, in the first LMR the sensitivity increase is proportionally much higher that that observed for the second, third and fourth LMR, with a lower increase. Moreover, the sensitivity increases non-linearly as the refractive index of the CRMOF, which is that of silica (see section 3), is approached.

\subsection{Sensitivity to coating refractive index variations}

In this subsection a theoretical analysis of the sensitivity to coating refractive index variations is done. The refractive index is composed of real and imaginary part. The real part is responsible for wavelength shift of the LMR, whereas the imaginary part is responsible for the depth of the LMR. 
Since the scope of this work is the analysis of the resonance wavelength, focus will be centered on the real part of the coating refractive index. However, prior to continue with the analysis of the real part or the coating refractive index it is necessary to prove the fact that the imaginary part is responsible for the depth of the LMR. Transmission spectra are obtained in Fig. 11 with a coating of thickness $333 \mathrm{~nm}$ and refractive index that of $\mathrm{TiO}_{2} / \mathrm{PSS}$ (Fig. 3a), but with different imaginary part refractive index values. The SMRI is 1.321 .

The increase in the imaginary part leads to a deeper LMR, whereas a decrease in the imaginary part leads to a not so deep LMR. Moreover, the LMR is divided into two local minima. This was observed experimentally with an $\operatorname{In}_{2} \mathrm{O}_{3}$ coating in [4], where it was explained that each of these minima are caused by coupling to a TE and a TM mode respectively. For the cases studied in this work the resonances are very close in the optical spectrum and it is not possible to distinguish the individual coupling. Therefore, a single attenuation band is observable.

Now, the effect of varying the real part of the coating refractive index will be studied in more detail. For the sake of simplicity we will refer to the real part of the coating refractive index as the coating refractive index.

First, the resonance wavelengths of LMRs are plotted in Fig. 12 as a function of the coating refractive index for two different thickness values of the thin-film: 236 and $1200 \mathrm{~nm}$. As imaginary part of the thin-film refractive index it has been chosen that of $\mathrm{TiO}_{2} / \mathrm{PSS}$ (see Fig. 3b). The SMRI is fixed to 1.321 . In this case, similarly to the analysis of section 4.2 , the number of LMRs obtained for a thickness of $1200 \mathrm{~nm}$ is higher than the number of LMRs obtained with a thickness of $236 \mathrm{~nm}$. In general, thick coatings support more LMRs than thin coatings under the same conditions (coating refractive index and surrounding media refractive index). 
Second, the plots of the LMRs are almost linear, which indicates that $\mathrm{S}_{\text {coating_index }}$ (the sensitivity to coating refractive index expressed as wavelength shift versus coating refractive index variation) remains constant for different refractive index values of the coating.

In addition to this, it can also be observed that $\mathrm{S}_{\text {coating_index }}$ increases progressively as we move towards the first LMR in both cases (236 nm and $1200 \mathrm{~nm}$ coating). In fact, if we compare the second LMR generated with the $236 \mathrm{~nm}$ coating with the seventh one obtained with the $1200 \mathrm{~nm}$ coating, both of them fit almost exactly, which proves that LMRs plotted as a function of the coating thickness occupy the same positions if they show the same $S_{\text {coating_index }}$ provided the surrounding medium refractive index remains constant.

In view of the difference between the results obtained with $236 \mathrm{~nm}$ and $1200 \mathrm{~nm}$ coatings, it is interesting to analyze $S_{\text {coating_index }}$ as a function of the thickness for a fixed SMRI of 1.321 (see Fig. 13a). In order to avoid the influence of wavelength, $\mathrm{S}_{\text {coating_index }}$ is evaluated as the thickness variation that leads to a resonance wavelength shift between 700 and $800 \mathrm{~nm}$. The same is true for the analysis of Fig. 13b, which will be analyzed later.

After seeing the results of Fig. 12 and Fig. 13a, it can be concluded that, in order to increase

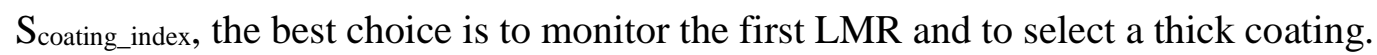

Two other important conclusions can be extracted from Fig. 13a. The first one is that $S_{\text {coating_index }}$ increases almost linearly for all LMRs. The second one is that there are no data available for thickness values higher than $200 \mathrm{~nm}$ in the case of the first LMR, and for values higher than $1000 \mathrm{~nm}$ for the second LMR. This indicates that LMRs shift to higher wavelengths than those monitored in the spectrum between 400 and $1500 \mathrm{~nm}$. Consequently, the advantage of increasing the thickness in terms of better $S_{\text {coating_index }}$ becomes a problem of detection of the LMR. 
This problem was not observed in Fig. 9. The reason is that for the studied range of coating refractive indices (1.5-2.1) and SMRIs (1.321-1.421) used in this work, the modification of the resonance wavelengths is not so abrupt as in the case of the studied thickness range $(0-1200 \mathrm{~nm})$. It is important to indicate that the values used for the study of the influence of thickness and refractive index of the coating, and SMRI are those typically used in experiments. In special cases other values could be used, but at least it allows us to conclude that it is more probable to obtain the first LMRs out of the spectral scope when we move to higher thickness values than when we move to higher coating refractive index or SMRI.

As it was explained before, the $S_{\text {coating_index }}$ observed in Fig. 13a for the first LMR shifts out of the studied scope for a thickness of $200 \mathrm{~nm}$. Thus, for thicker coatings, the best monitorizable $\mathrm{S}_{\text {coating_index }}$ within the studied range is that of second LMR, which increases linearly until a thickness of $1000 \mathrm{~nm}$. If we continue increasing the thickness of the coating over $1000 \mathrm{~nm}$, the best $\mathrm{S}_{\text {coating_index }}$ within the studied range is now that of the third LMR which increases also linearly, and so on. Consequently, the increase of $S_{\text {coating_index }}$ as a function of thickness becomes a decrease at certain points when low order LMRs shift progressively out of the monitorizable spectrum.

The evolution of the resonance wavelengths of the LMRs generated with a $600 \mathrm{~nm}$ coating is represented in Fig. 14 as a function of the coating refractive index for two different SMRIs: 1.321 (i.e. that of water) and 1.421. It is clear that $\mathrm{S}_{\text {coating_index }}$ is improved for all LMRs when the SMRI is increased.

In order to obtain a better analysis, the evolution of $\mathrm{S}_{\text {coating_index }}$ as function of the SMRI, is plotted for a coating thickness of $600 \mathrm{~nm}$ in Fig. 13b. As in Fig. 13a, there is also an increase as a function of the parameter of the $\mathrm{x}$ axis (in Fig. 13a it is the thickness and Fig. 13b it is the 
SMRI), but in this case the increase is non-linear. There is a slight increase of the plot slope as the refractive index of silica is approached. In the second LMR this increase is proportionally higher than in the third one.

\subsection{Sensitivity to surrounding medium refractive index variations (SMRI)}

In this subsection it is presented a theoretical analysis of the sensitivity to SMRI variations.

First, the resonance wavelengths of LMRs are plotted in Fig. 15 as a function of the SMRI for two different thickness values of the coating: 48 and $1200 \mathrm{~nm}$. The coating refractive index is that of $\mathrm{TiO}_{2} / \mathrm{PSS}$. Similar to the analysis of section 4.2 and 4.3, the number of LMRs obtained at a thickness of $1200 \mathrm{~nm}$ is higher than the number of LMRs obtained with a thickness of $48 \mathrm{~nm}$. The explanation is that a thick coating contains a higher number of lossy modes than a thin coating under the same conditions (coating refractive index and surrounding media refractive index).

In contrast to the analysis of the coating thickness and coating refractive index, the plots of the LMRs are non-linear (this is especially observed in the first LMR of the $48 \mathrm{~nm}$ thick coating, but could be also observed for the rest of LMRs in the $1200 \mathrm{~nm}$ thick coating with a zoom in the

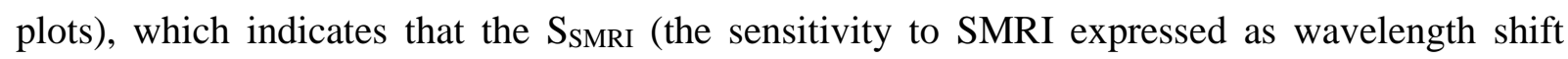
versus SMRI variation) does not remain constant for different SMRI values. In fact, the $\mathrm{S}_{\mathrm{SMRI}}$ increases progressively as the refractive index of the optical fiber is approached.

Another interesting conclusion is that, unlike in the analysis of subsections 4.2 and 4.3 , there is no superposition of the LMRs plotted as a function of the SMRI for the same coating refractive index. In fact the $\mathrm{S}_{\mathrm{SMRI}}$ is better for thinner coatings. Thus, in order to obtain an optimal sensitivity, the best choice is to monitor the first LMR of a thin coating. 


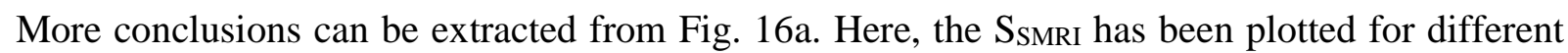
thickness values of the coating. The coating refractive index is that of $\mathrm{TiO}_{2} / \mathrm{PSS}$. In view of the flatness of the curves of Fig. 15, it is necessary to modify in this subsection the criterion used for the sensitivity in subsections 4.2 and 4.3. Now the $\mathrm{S}_{\mathrm{SMRI}}$ is the quotient between the wavelength shift experimented in the range between 1.321 and 1.421

In Fig. 16a it is clearly appreciated that the $\mathrm{S}_{\mathrm{SMRI}}$ increase as a function of thickness is linear for all LMRs, something that was also observed in Figs. 13a when $S_{\text {coating_index }}$ as a function of thickness was analyzed. The similarity between Fig. 16a and Fig. 13a helps to explain that there are no data available for thickness values higher than $600 \mathrm{~nm}$ in the case of the first LMR. This indicates that the LMR shifts to longer wavelengths than those monitored in the spectrum between 400 and $1500 \mathrm{~nm}$. Consequently, the advantage of increasing the thickness in terms of better $\mathrm{S}_{\text {SMRI }}$ becomes a problem in the detection of the LMR. The increase of $\mathrm{S}_{\text {SMRI }}$ observed for the first LMR within the range 400-1500 nm is not monitorizable for a thickness of $600 \mathrm{~nm}$. For thicker coatings the best sensitivity is that of second LMR (lower than the $\mathrm{S}_{\text {SMRI }}$ of the first LMR), which also increases linearly.

In Fig. 17 the evolution of the resonance wavelengths of the LMRs generated with a $600 \mathrm{~nm}$ coating is represented as a function of the SMRI for two coating refractive indices: 1.55 and 2.1. Like in Fig. 15, there is no superposition of the LMRs plotted as a function of the SMRI for the same coating thickness. In addition to this, there is an increase of $\mathrm{S}_{\text {SMRI }}$ as we move to the first LMR, which had been observed in subsections 4.2, 4.3 and now in subsection 4.4.

For a better analysis, the $\mathrm{S}_{\mathrm{SMRI}}$ has been plotted in Fig. $16 \mathrm{~b}$ for different coating refractive indices and a coating thickness of $600 \mathrm{~nm}$. The increase in the sensitivity can be observed for all LMRs. However, similarly to what happened in Fig. 9a, the rate of increase is non-linear and decays at 
longer wavelengths. Moreover, in this case it seems that it achieves a maximum value. An additional disadvantage that prevents to move to higher coating refractive indices is that the first LMR is out of scope for coating refractive indices higher than 2. For these cases the less sensitive second LMR should be monitored.

\subsection{Final remarks}

In Table I there is summary of the rules that can be used to design optimum LMR based devices. The parameters analyzed are the most important ones in terms of design of LMR based sensors. These rules have been extracted from materials that present conditions for LMR in a wide wavelength range. According to Fig. 3, this is the case for the materials analyzed in this work: PAH/PAA and $\mathrm{TiO}_{2} / \mathrm{PSS}$. However, if other materials are used, attention must be paid if there are regions in the spectrum analyzed where the conditions for SPRs are met. This is the case of ITO. In [1] it is proved that the LMR generated with ITO coated CRMOF is not linearly shifted as a function of thickness. This is a contradiction with the results of Fig. 8 in this work. The reason is that the presence of a region where the conditions for formation of SPR are met prevents the LMR to be shifted to higher wavelengths.

\section{CONCLUSIONS}


Some rules have been obtained for adequate design of sensing devices based on deposition of a thin-film on a cladding removed multimode optical fiber.

The sensing mechanism is based on the detection of the central wavelength of lossy mode resonances (LMR) generated only for specific conditions (i.e. the real part of the thin-film permittivity is positive and higher in magnitude than both its own imaginary part and the material surrounding the thin-film)

The key parameters for the design are the thickness and real part of the refractive index of the thin film, and the refractive index of the surrounding medium. The imaginary part of the thinfilm refractive index is only responsible for the generation of dual resonances and for the depth of the resonance, but not for the wavelength shift.

According to the results obtained, the sensitivity is constant or increases as a function of the three parameters studied with some particularities. However, when the value of the key parameters is increased there are certain points where the most sensitive LMR (first LMR) is out of scope in the spectral range analyzed. In these cases it is necessary to monitor the second, the third and so on less sensitive LMRs.

\section{ACKNOWLEDGMENTS}

This work was supported by the Spanish Ministry of Education and Science-FEDER TEC201017805 and Government of Navarra research grants. Special thanks to FIDENA for the utilization of the SEM. 


\section{REFERENCES}

1. I. Del Villar, C. R. Zamarreño, M. Hernaez, F. J. Arregui, I. R. Matias, "Lossy mode resonance generation with indium tin oxide coated optical fibers for sensing applications," J. Lightwave Technol. 28, 111 (2010).

2. C. R. Zamarreño, M. Hernáez, I. Del Villar, I. R. Matias and F. J. Arregui, “Tunable humidity sensor based on ITO-coated optical fiber," Sens. Actuators B 146, 414-417 (2010).

3. M. Hernaez, I. Del Villar, C. M. Zamarreño, F. J. Arregui, I. R. Matias, “Optical fiber refractometers based on lossy mode resonances supported by TiO2 coatings" Appl. Opt. 49, 3980-3985 (2010)

4. I. Del Villar, C. R. Zamarreño, M. Hernáez, P. Sánchez, Carlos F. Valdivielso, F. J. Arregui and I. R. Matías "Generation of lossy mode resonances by deposition of high-refractiveindex coatings on uncladded multimode optical fibers," J. Opt. 12, 095503 (7pp), (2010).

5. C. R. Zamarreño, M. Hernáez, I. Del Villar, I. R. Matías and F. J. Arregui "Optical fiber pH sensor based on lossy-mode resonances by means of thin polymeric coatings," Sens. Actuators B 155, 290-297 (2011).

6. T. E. Batchman and G. M. McWright, "Mode coupling between dielectric and semiconductor planar waveguides,” IEEE J. Quantum Electron. 18, 782-788 (1982).

7. M. Marciniak, J. Grzegorzewski, M. Szustakowski, “Analysis of lossy mode cut-off conditions in planar waveguides with semiconductor guiding layer" IEE Proceedings J. 140, 247-252 (1993).

8. D. Razansky, P. D. Einziger and D. R. Adam, "Broadband absorption spectroscopy via excitation of lossy resonance modes in thin films,” Phys. Rev. Lett. 95, 018101 (2005). 
9. F. Yang and J. R. Sambles, "Determination of the optical permittivity and thickness of absorbing films using long range modes,” J. Mod. Opt. 44, 1155-1163 (1997).

10. A. K. Sharma and B. D. Gupta, "On the sensitivity and signal to noise ratio of a step-index fiber optic surface plasmon resonance sensor with bimetallic layers," Opt. Comm. 245, 159169 (2005).

11. R. C. Jorgenson and S. S. Yee, “A fiber-optic chemical sensor based on surface Plasmon resonance," Sens. Actuators B 12, 213-220 (1993).

12. R. Ota, S. Seki, M. Ogawa, T. Nishide, A. Shida, M. Ide and Y. Sawada, "Fabrication of indium-tin-oxide films by dip coating process using ethanol solution of chlorides and surfactants," Thin Solid Films, 411, $42-45$ (2002).

13. G. Decher, "Fuzzy nanoassemblies: toward layered polymeric multicomposites," Science 277, 1232-1237 (1997).

14. Y. Xu, N. Barrie Jones, J. C. Fothergill and C. D. Hanning, "Analytical estimates of the characteristics of surface Plasmon resonance fibre optic sensors," J. Mod. Opt. 47, 1099$1110(2000)$.

15. G. P. Agrawal, "Nonlinear fiber optics” p. 8., (3rd ed., Academic Press: New York, 2001)

16. R. A. Synowicki, "Spectroscopic ellipsometry characterization of indium tin oxide film microstructure and optical constants,” Thin Solid Films 313-314, 394-397 (1998). 


\section{Figure captions}

Fig. 1: SEM image of a) $\mathrm{TiO}_{2} / \mathrm{PSS}$ coated optical fiber core with coating thickness $1200 \mathrm{~nm}, \mathrm{~b}$ ) PAH/PAA coated optical fiber core with coating thickness $1000 \mathrm{~nm}$.

Fig. 2: Experimental setup with the light source, the detector and the optical fiber with the coated region. Fig. 3: Refractive index dispersion curves of $\mathrm{TiO}_{2} / \mathrm{PSS}$ and PAH/PAA.

Fig. 4: Spectral response obtained as a function of thickness for $\mathrm{TiO}_{2} / \mathrm{PSS}$ coated CRMOF (the SMRI is 1.321): (a) theoretical, (b) experimental.

Fig. 5: Spectral response obtained as a function of thickness for PAH/PAA coated CRMOF (the SMRI is 1.321): (a) theoretical, (b) experimental.

Fig. 6: Transmission spectra for two different thickness values (333 and $1165 \mathrm{~nm}$ ) of $\mathrm{TiO}_{2} / \mathrm{PSS}$ coated CRMOF (the SMRI is 1.321): (a) theoretical, (b) experimental.

Fig. 7: Transmission spectra for two different thickness values (750 and $1200 \mathrm{~nm}$ ) of PAH/PAA coated CRMOF (the SMRI is 1.321): (a) theoretical, (b) experimental.

Fig. 8: LMR wavelength as function of coating thickness for two different materials: PAH/PAA and $\mathrm{TiO}_{2} / \mathrm{PSS}$. The SMRI is 1.321 (water). Simulation data: continuous line. Experimental data: squares.

Fig. 9: $S_{\text {thickness }}$ (sensitivity to variations of coating thickness expressed as wavelength shift vs thickness variation $\mathrm{nm} / \mathrm{nm}$ ) as a function of: a) coating refractive index (the SMRI is 1.321 ), b) surrounding medium refractive index (the coating refractive index is that of $\mathrm{TiO}_{2} / \mathrm{PSS}$ in Fig. 3).

Fig. 10: LMR wavelength as a function of coating thickness for two different surrounding medium refractive indices: 1.321 (water) and 1.421. Coating refractive index: $\mathrm{TiO}_{2} / \mathrm{PSS}$ in Fig. 3.

Fig. 11: Transmission spectra for different refractive indices. The real part is that of $\mathrm{TiO}_{2} / \mathrm{PSS}$. The imaginary part varies from 0.004 to 0.04 in steps of 0.004 . The SMRI is 1.321 (water). Coating thickness: $333 \mathrm{~nm}$.

Fig. 12: LMR wavelength as a function of the coating refractive index for two different coating thickness values: 236 and $1200 \mathrm{~nm}$. The SMRI is 1.321 (water). 
Fig. 13: $\mathrm{S}_{\text {coating_index }}$ (sensitivity to variations of coating refractive index expressed as wavelength shift vs refractive index $\mathrm{nm} / \mathrm{RIU}$ ) as a function of: a) coating thickness (the SMRI is 1.321 ), b) surrounding medium refractive index (the coating thickness is $600 \mathrm{~nm}$ ).

Fig. 14: LMR wavelength as a function of coating refractive index for two different surrounding medium refractive indices: 1.321 (water) and 1.421. Coating thickness: $600 \mathrm{~nm}$.

Fig. 15: LMR wavelength as a function of the surrounding medium refractive index for two different coating thickness values: 48 and $1200 \mathrm{~nm}$. Coating refractive index: $\mathrm{TiO}_{2} / \mathrm{PSS}$ in Fig. 3.

Fig. 16: $\mathrm{S}_{\mathrm{SMRI}}$ (sensitivity to variations of SMRI expressed as wavelength shift vs refractive index variation $\mathrm{nm} / \mathrm{RIU}$ ) as a function of: a) coating thickness (the coating refractive index is that $\mathrm{TiO}_{2} / \mathrm{PSS}$ in Fig. 3), b) coating refractive index (the coating thickness is $600 \mathrm{~nm}$ ).

Fig. 17: LMR wavelength as function of the surrounding medium refractive index for two different coating refractive indices: 1.55 and 2. Coating thickness: $600 \mathrm{~nm}$. 


\section{Figures}

Fig. 1
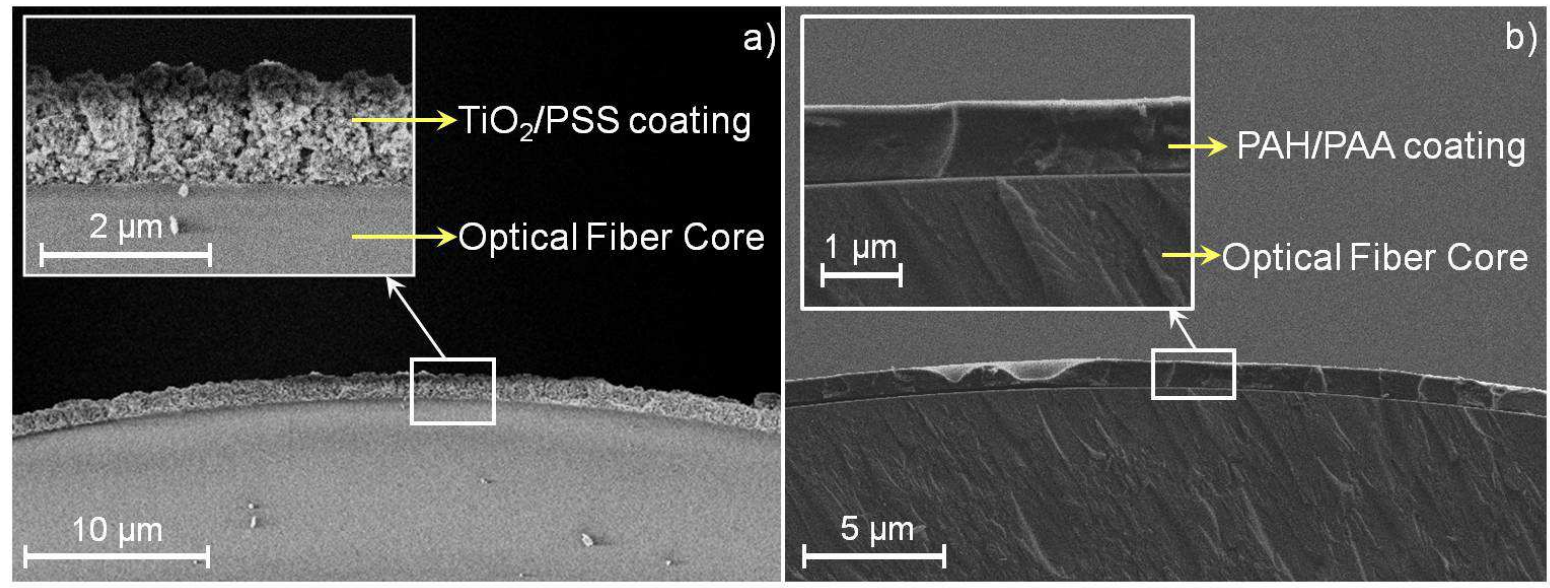
Fig. 2

\section{a) Experimental Set-up}

White Light Source

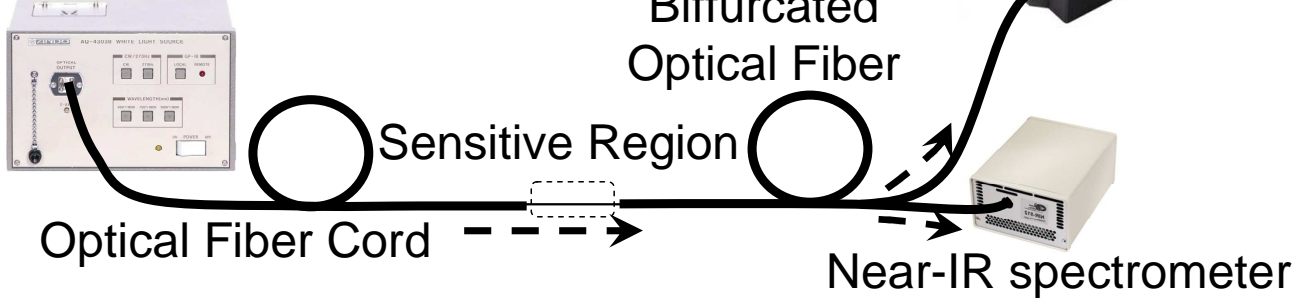

b) Sensitive Region (meridional section)

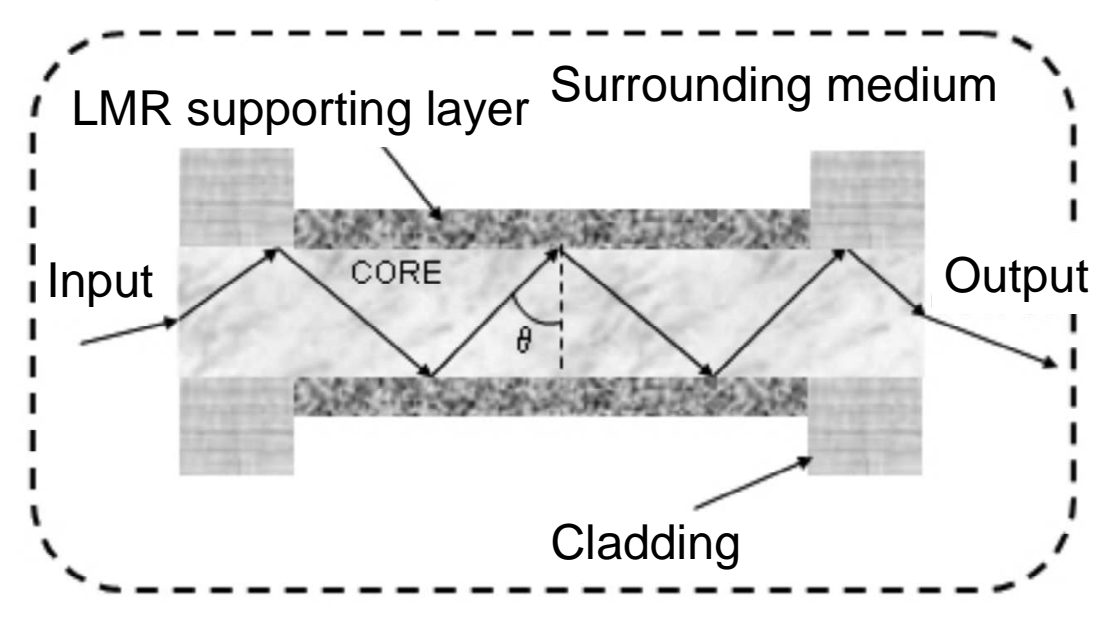


Fig. 3
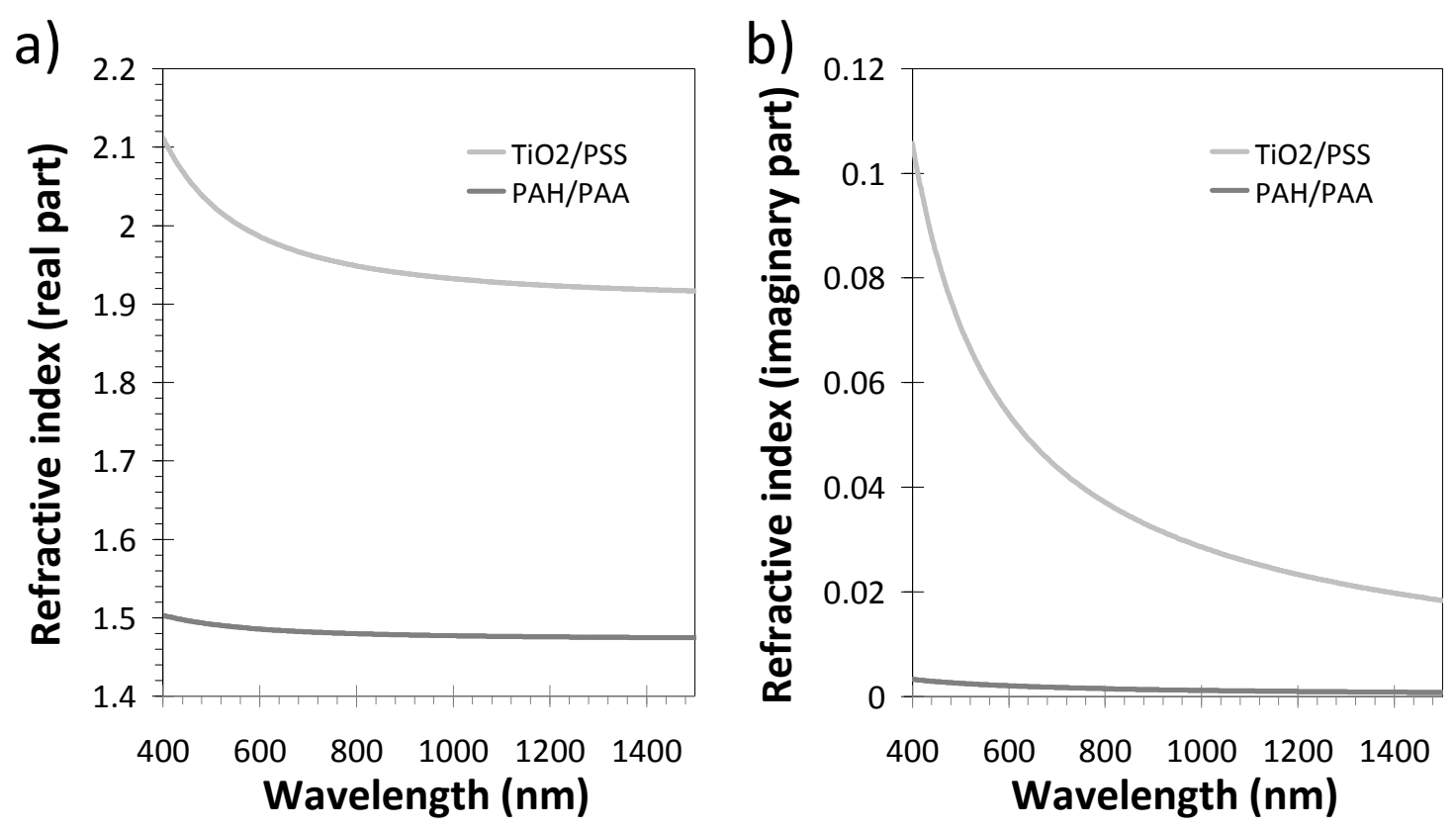
Fig. 4

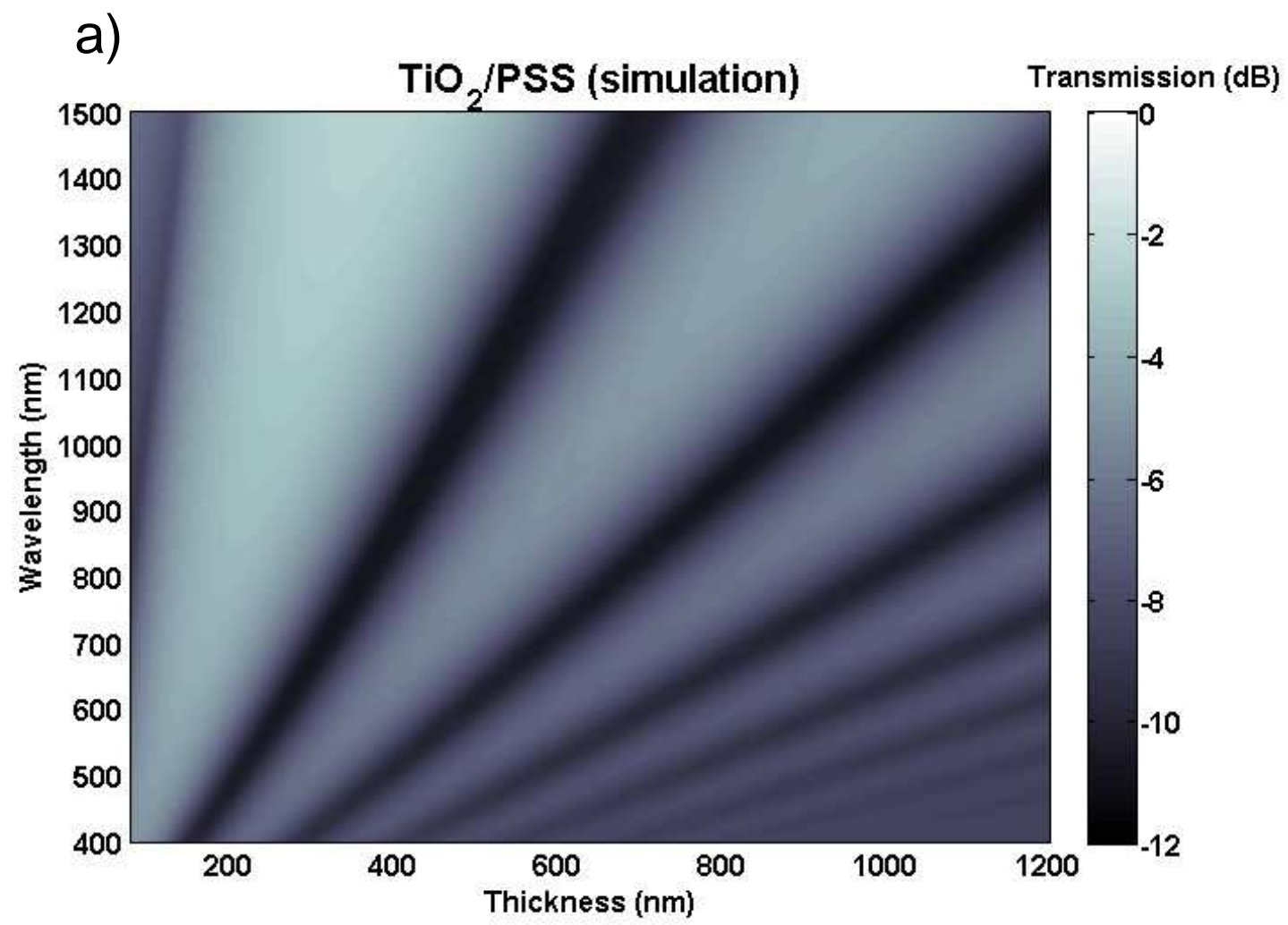

b)

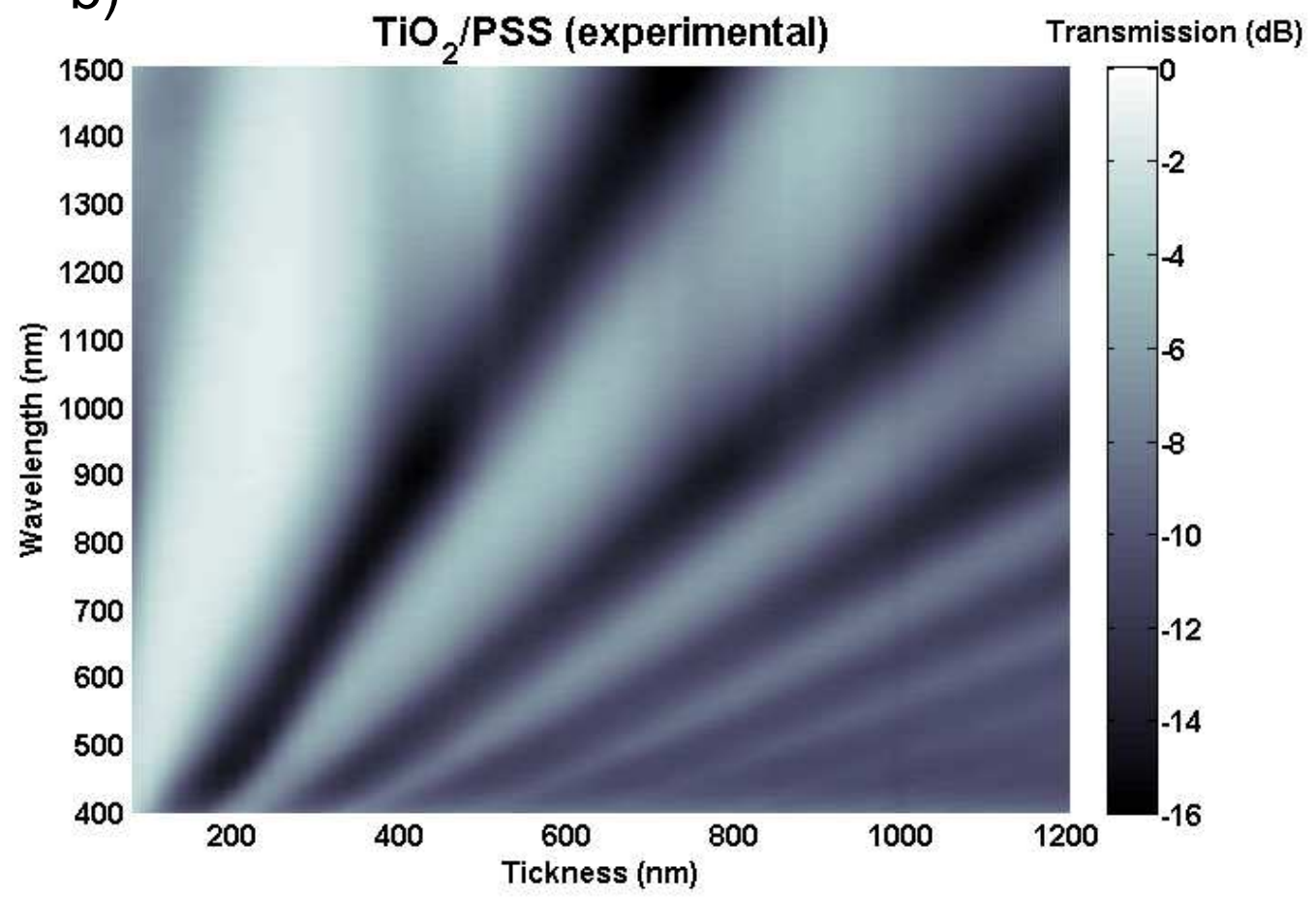


Fig. 5

a)

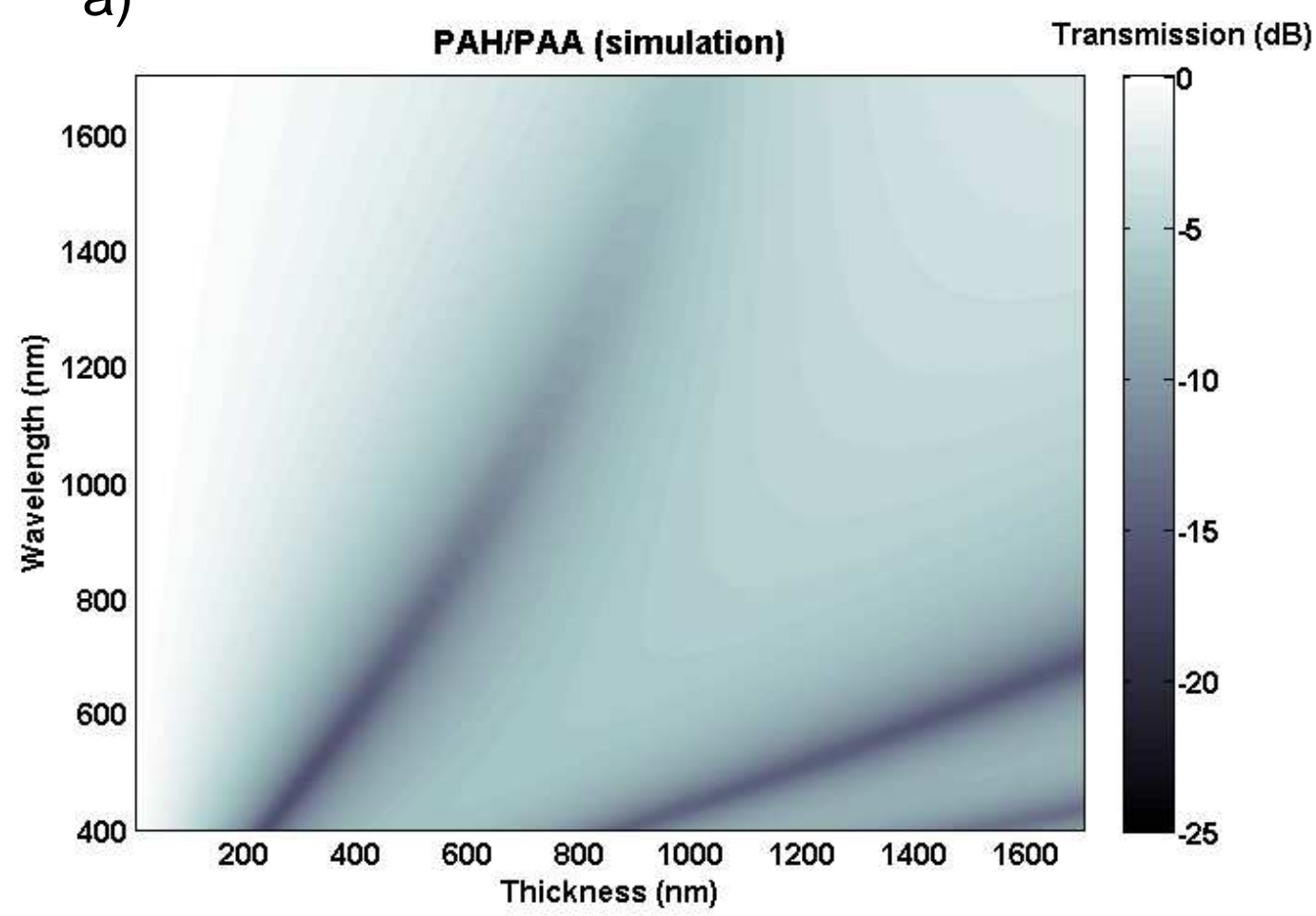

b)

PAHIPAA (experiment)

Transmission (dB)

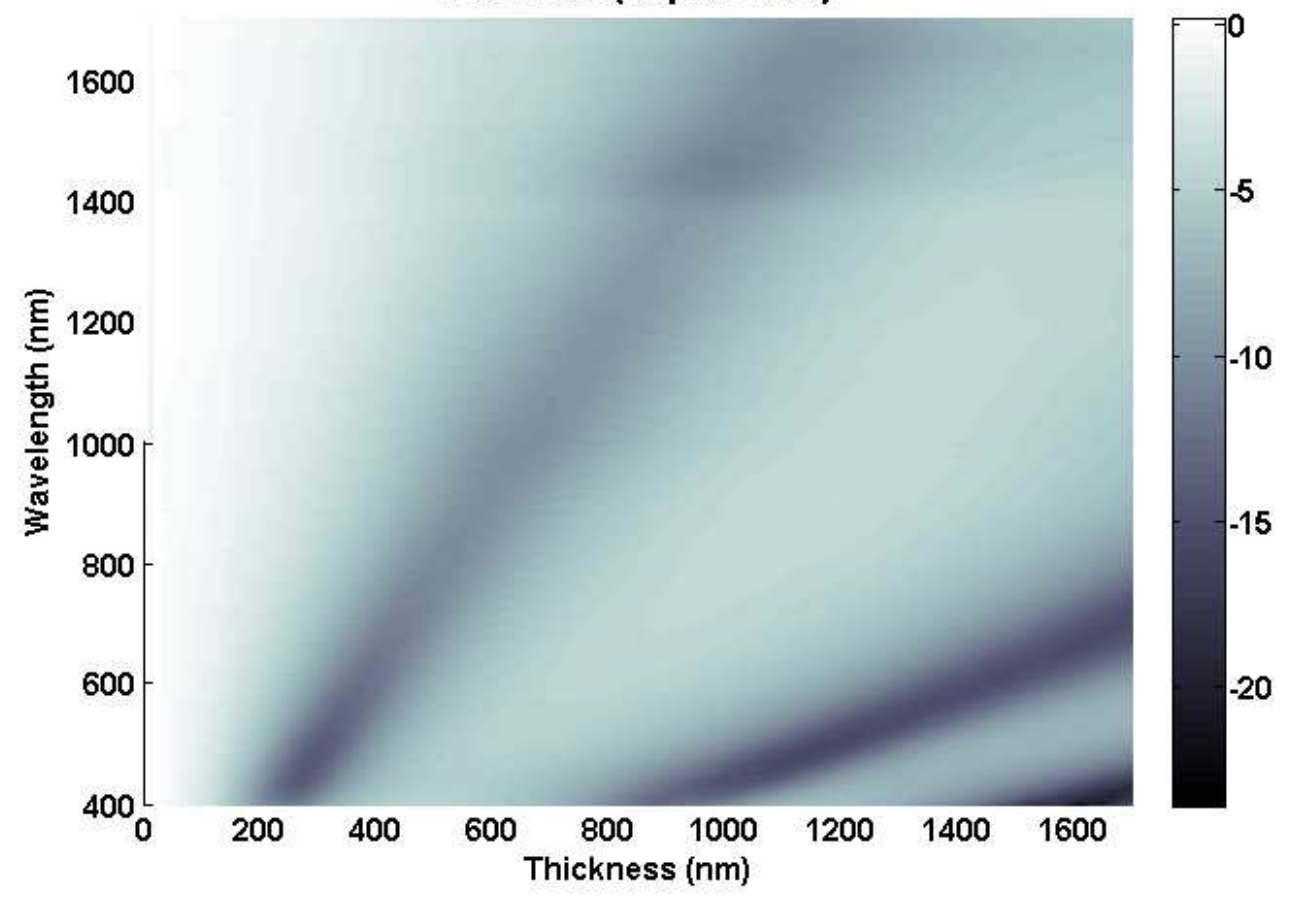


Fig. 6
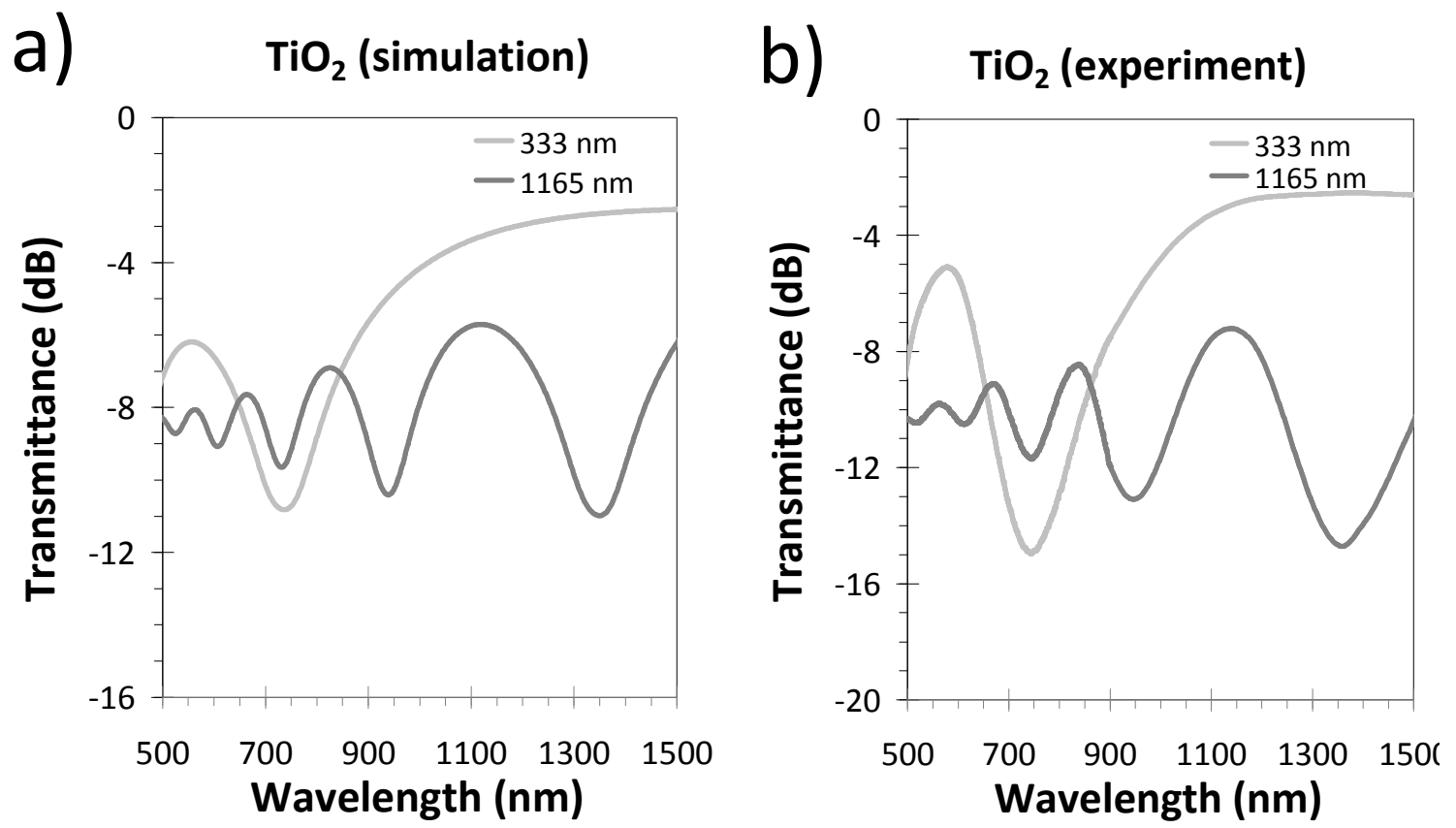
Fig. 7
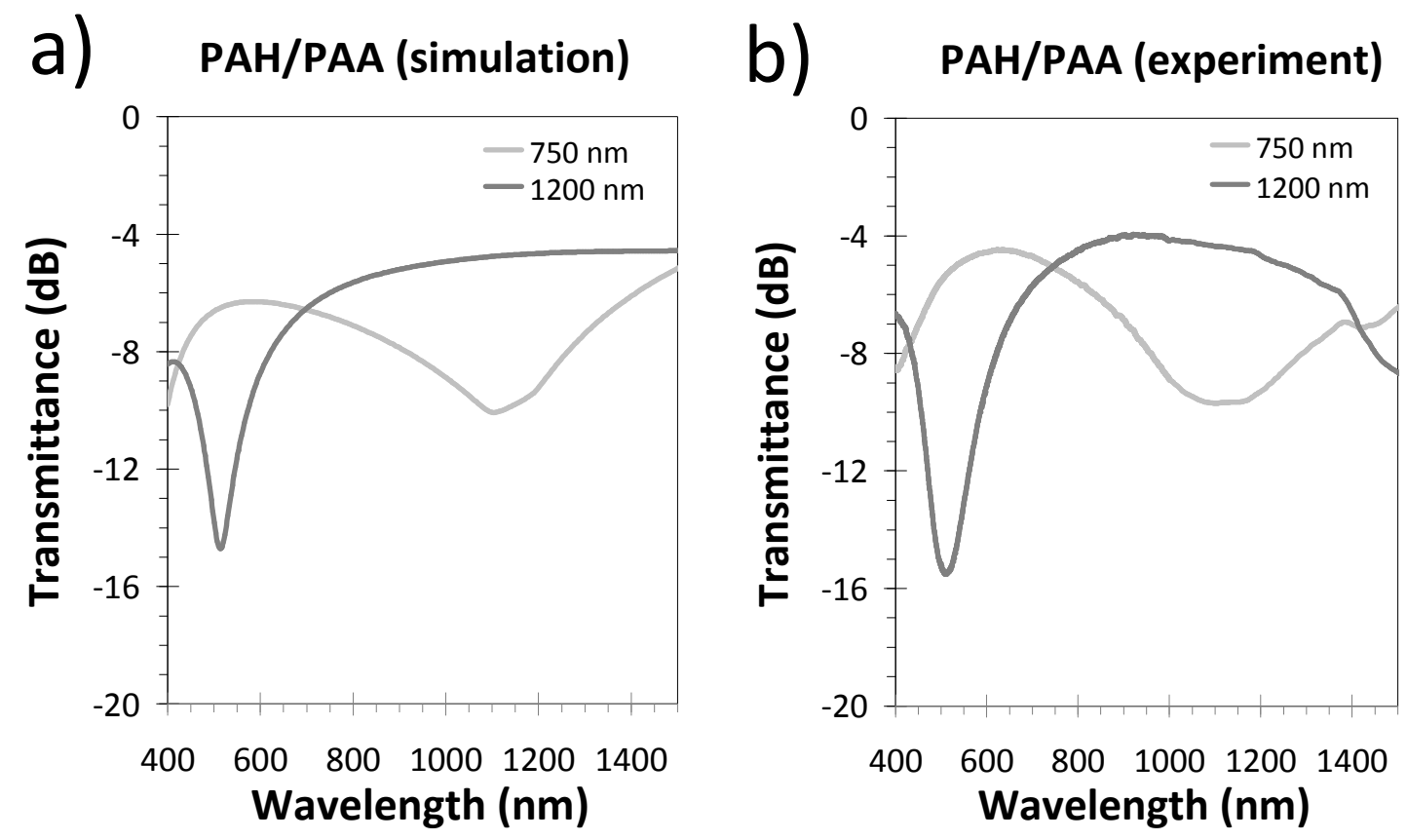
Fig. 8

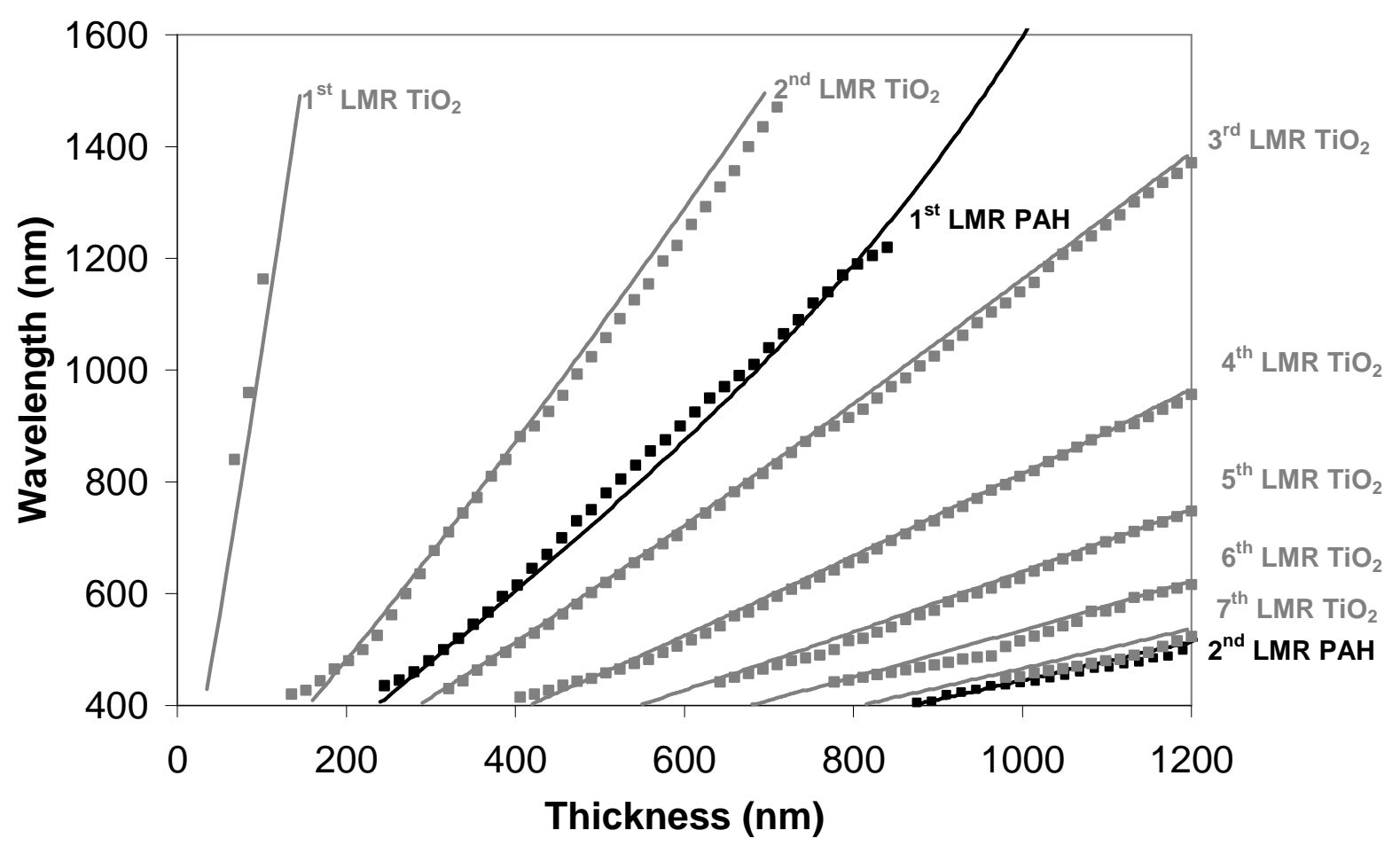


Fig. 9

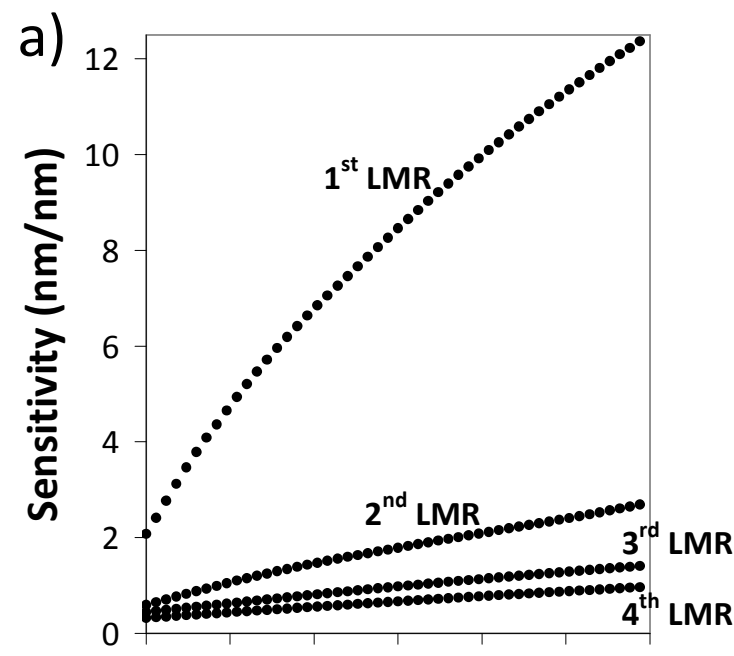

$\begin{array}{lllllll}1.5 & 1.6 & 1.7 & 1.8 & 1.9 & 2.0 & 2.1\end{array}$ Coating refractive index b)

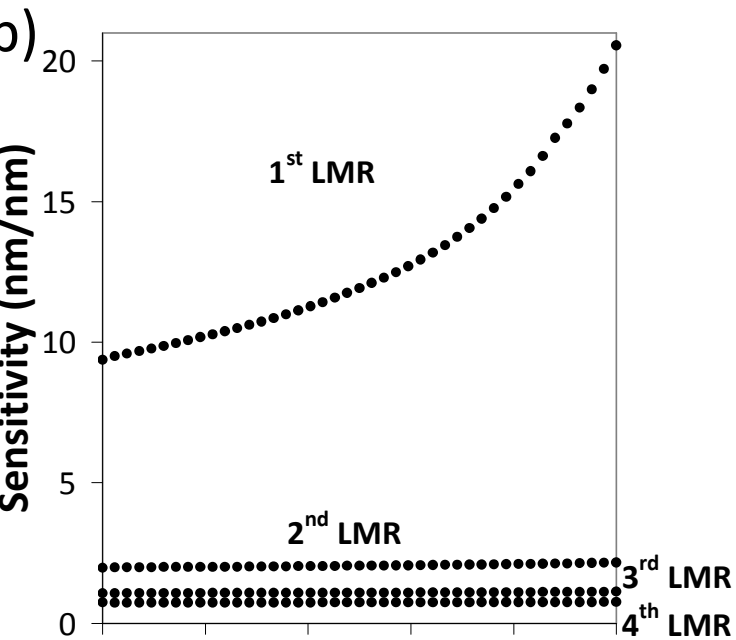

$\begin{array}{llllll}1.32 & 1.34 & 1.36 & 1.38 & 1.40 & 1.42\end{array}$ Surrounding medium ref index 
Fig. 10

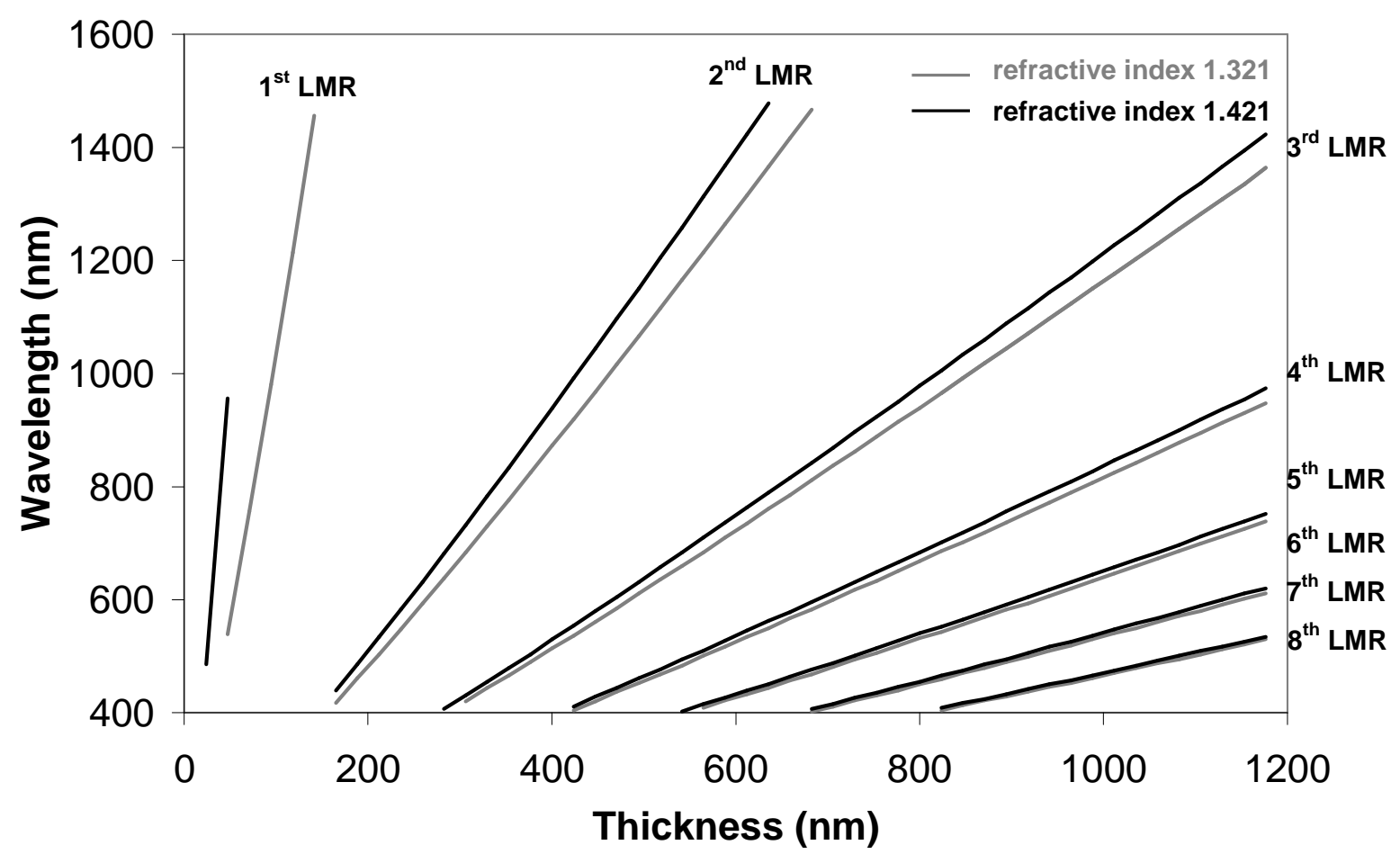


Fig. 11

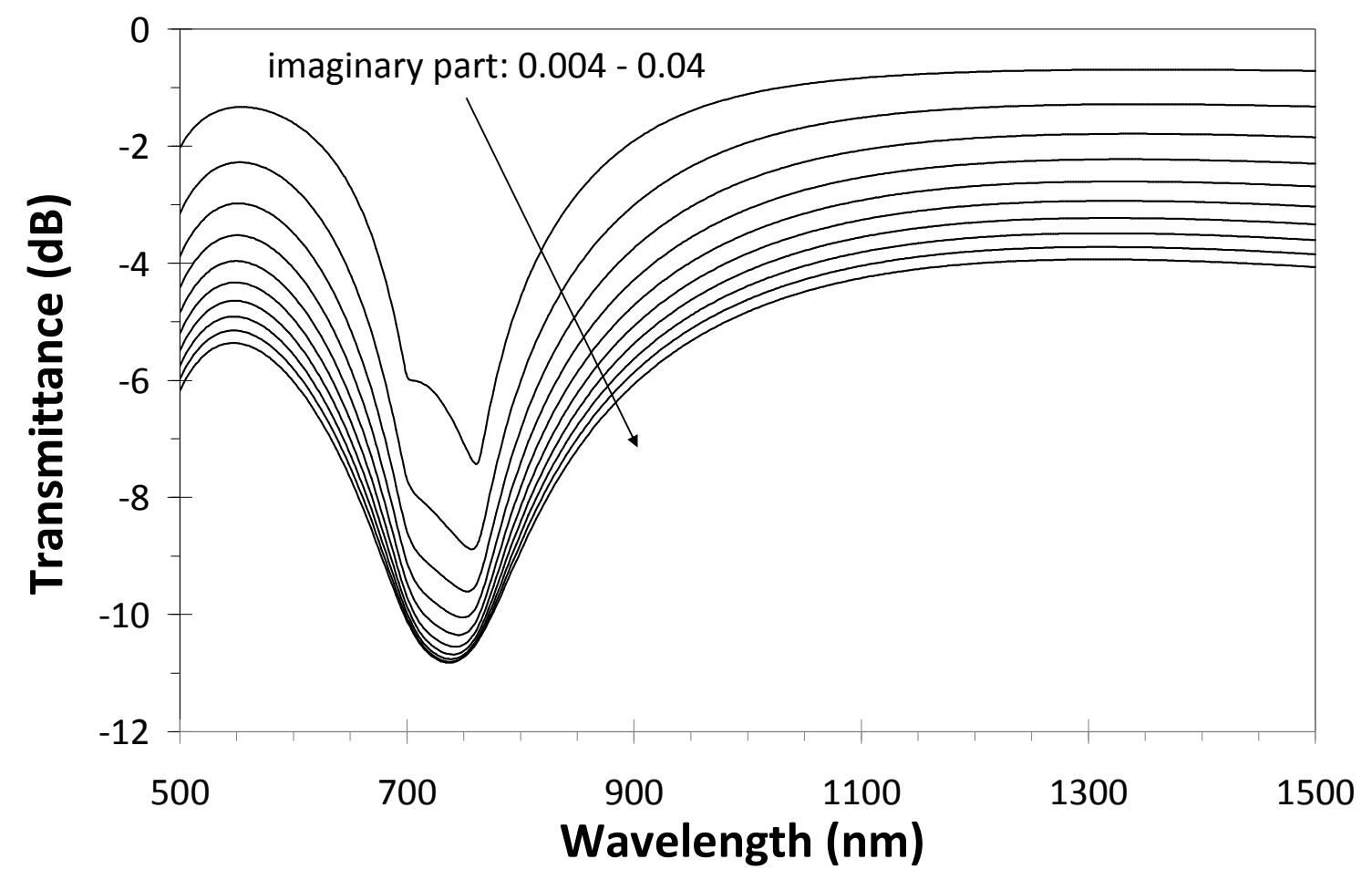


Fig. 12

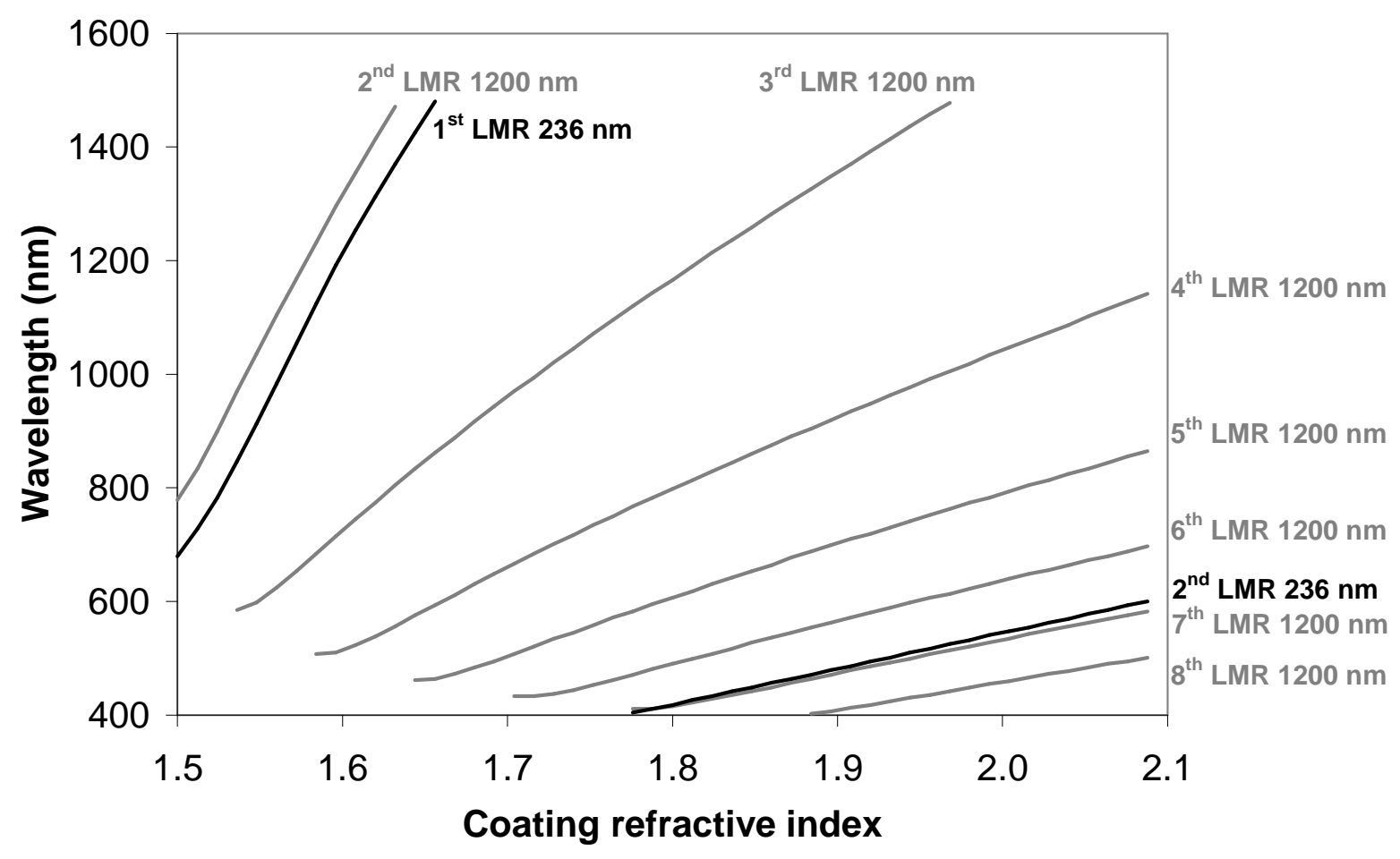


Fig. 13
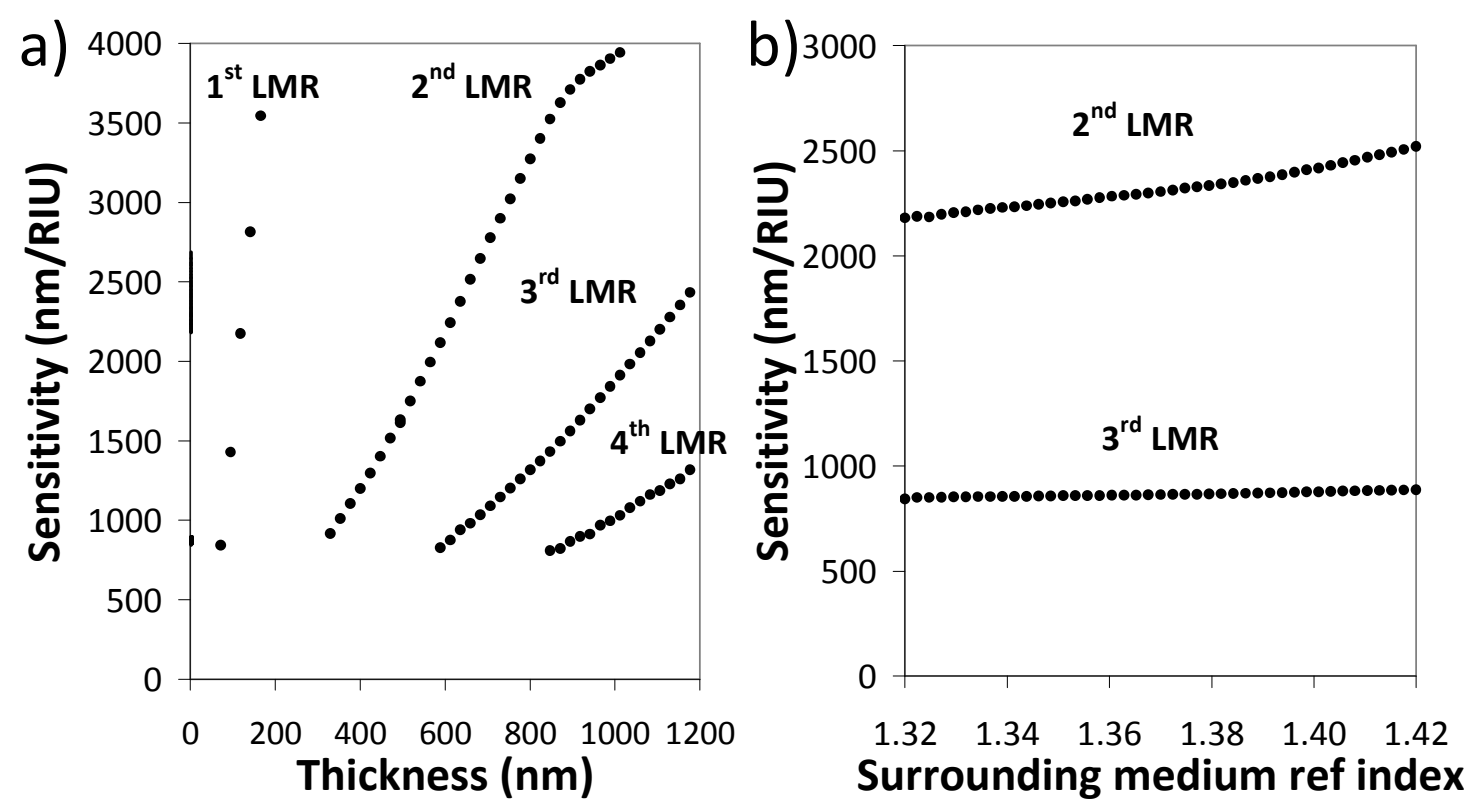
Fig. 14

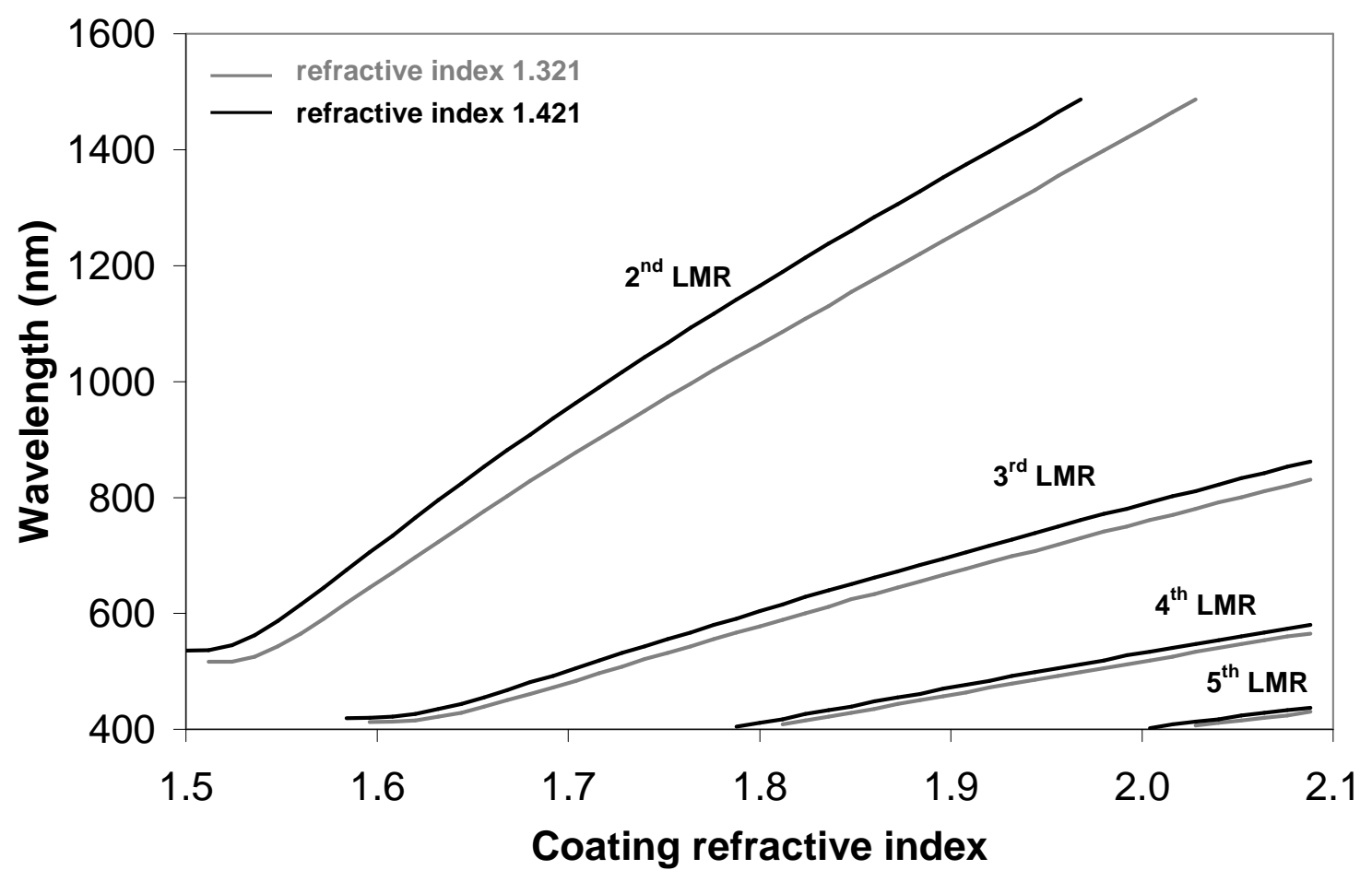


Fig. 15

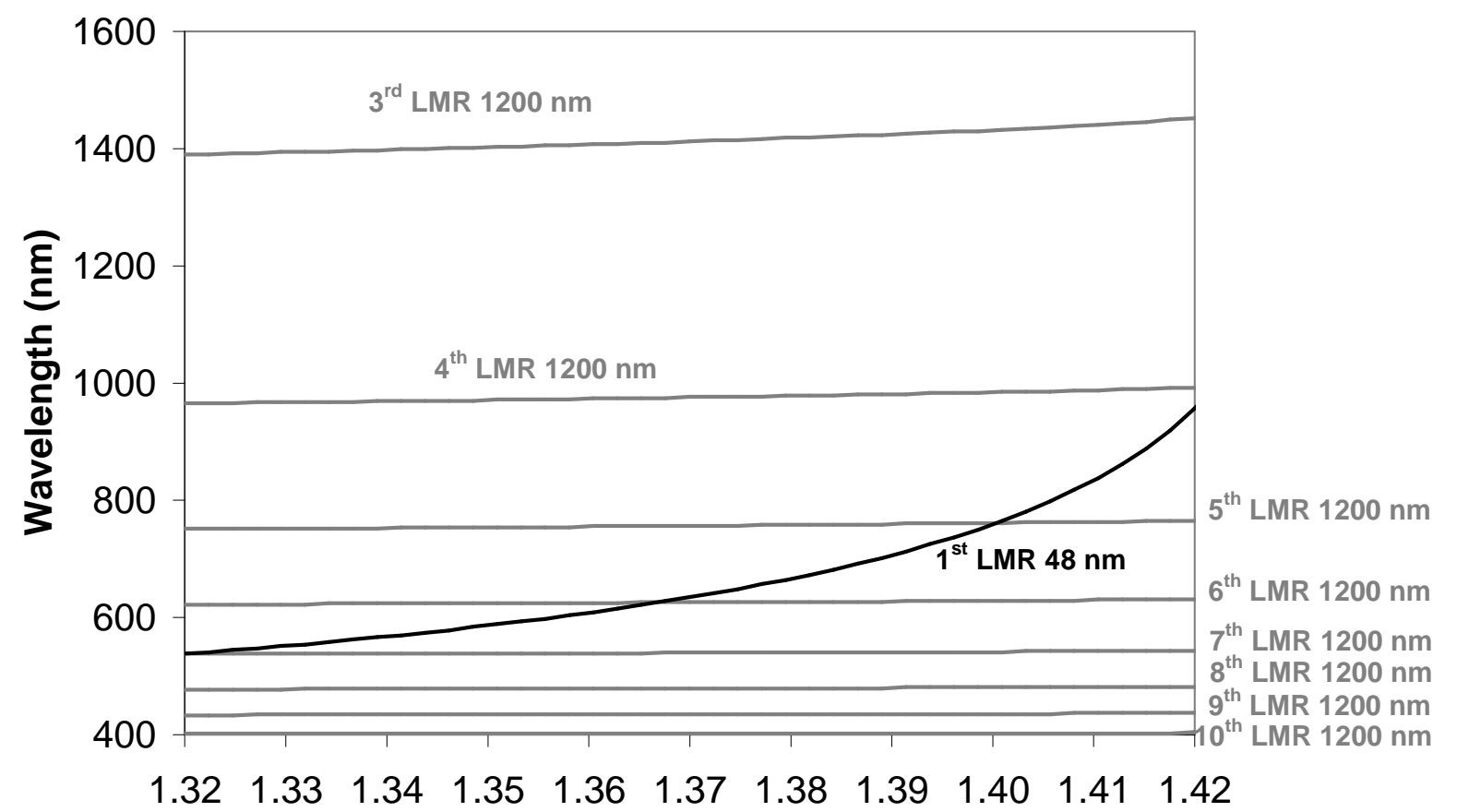

Surrounding medium refractive index 
Fig. 16
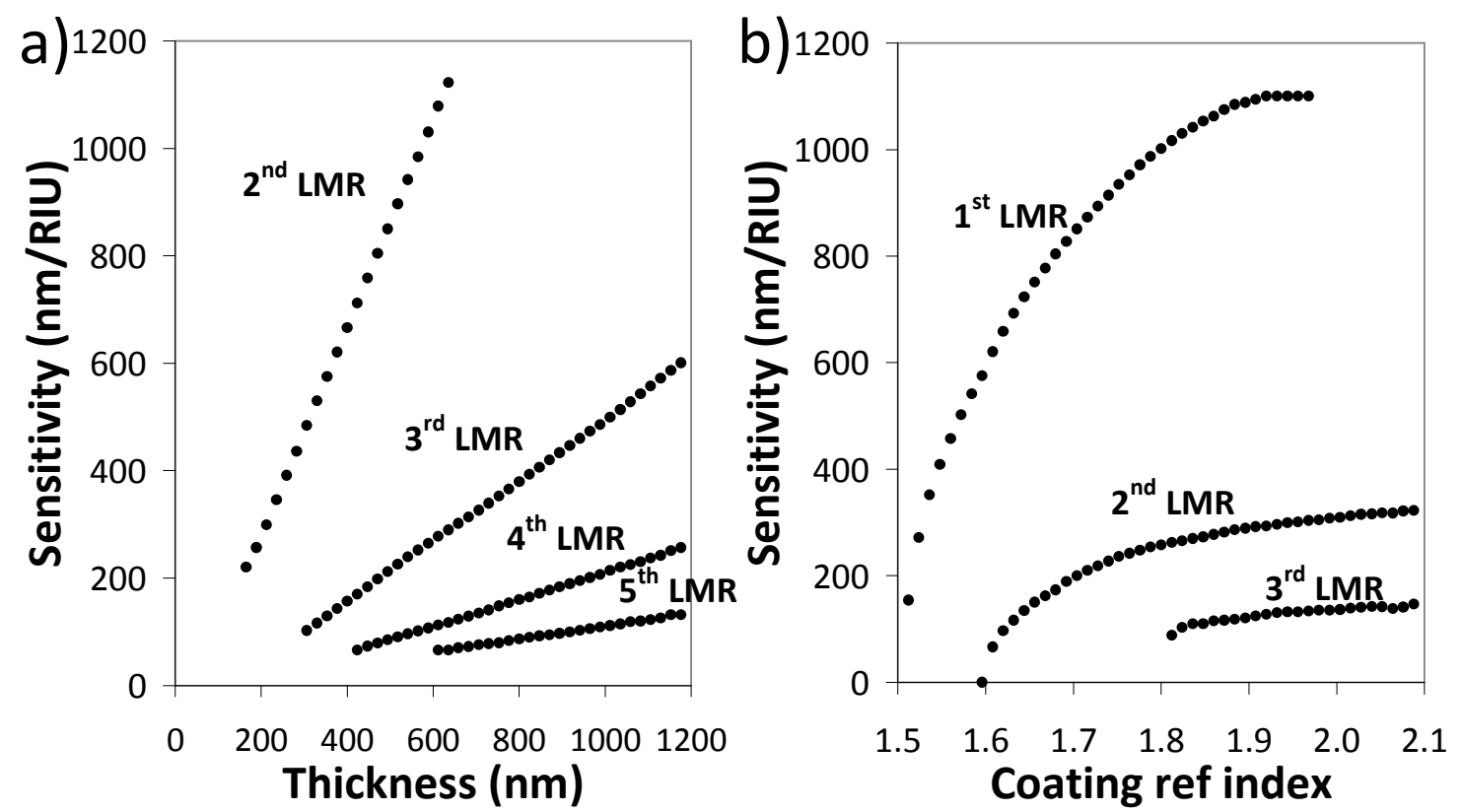
Fig. 17

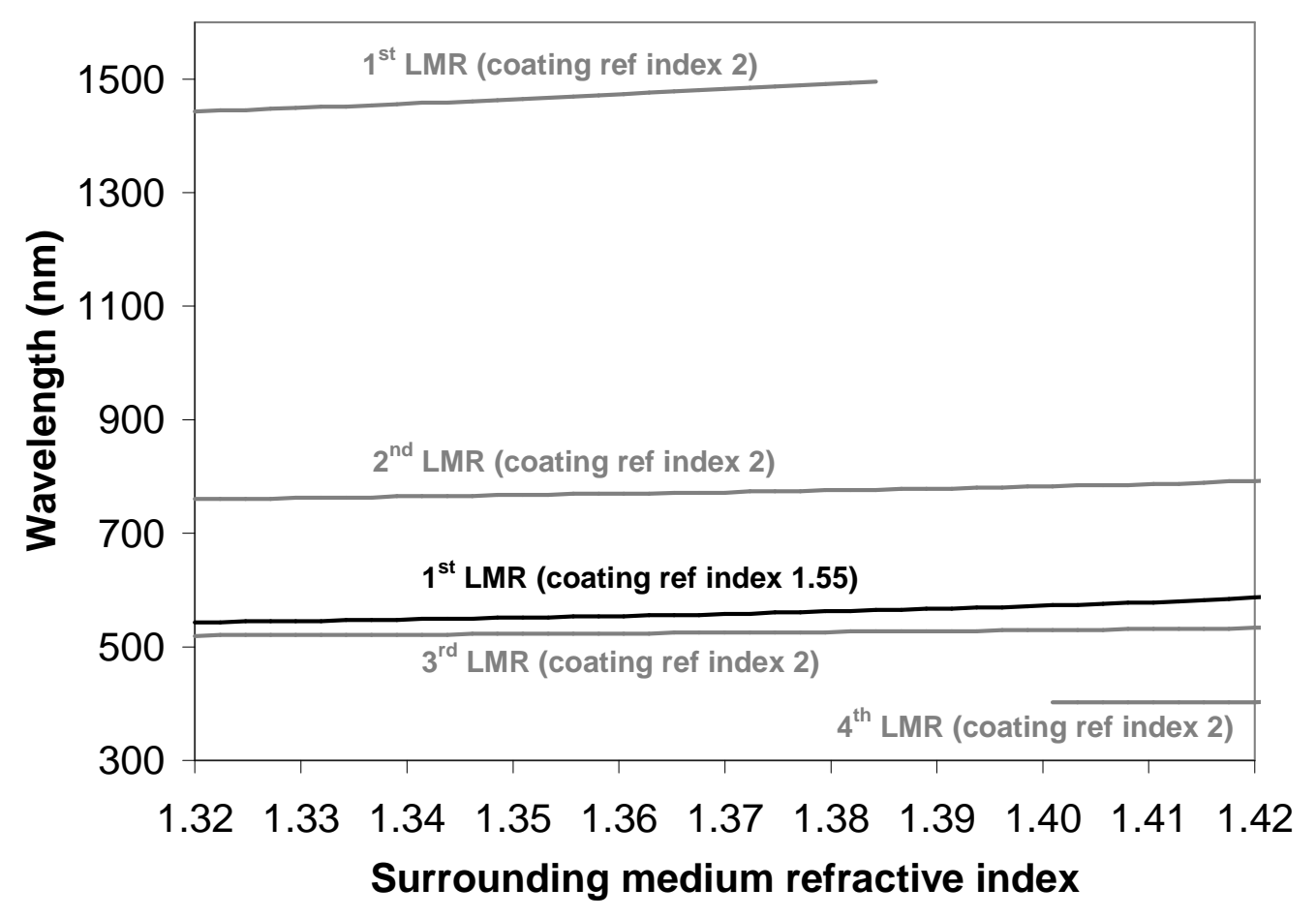




\section{Table captions}

Table 1: Rules for sensitivity, expressed as wavelength shift vs variation of a parameter, for the different parameters analyzed in this work. 
Table 1

\begin{tabular}{|c|c|c|c|}
\hline & \multicolumn{3}{|c|}{ Sensitivity } \\
\hline & Sthickness & S $_{\text {coating_index }}$ & SSMRI \\
\hline Coating thickness & constant & increasing linearly & increasing linearly \\
\hline Coating refractive index & $\begin{array}{l}\text { Increasing non-linearly } \\
\text { (decreasing slope) }\end{array}$ & constant & $\begin{array}{l}\text { Increasing non-linearly } \\
\text { (decreasing slope) }\end{array}$ \\
\hline SMRI & $\begin{array}{c}\text { Increasing non-linearly } \\
\text { (increasing slope) } \\
\text { More separation between } \\
\text { LMRs }\end{array}$ & $\begin{array}{c}\text { Increasing non-linearly } \\
\text { (increasing slope) } \\
\text { More separation between } \\
\text { LMRs }\end{array}$ & increasing \\
\hline
\end{tabular}

